\title{
Matrix Metalloproteinases and Their Tissue Inhibitors in Patients with Nonalcoholic Fatty Liver Disease
}

\author{
Tatyana Krolevets, Maria Livzan and Marina Kolbina \\ Department of Therapy with the Course of Occupational (Professional) Diseases, the Ministry of Health of the Russian Federation, \\ Omsk State Educational Institution of Higher Vocational Education “Omsk State Medical University”, Omsk 644123, Russia
}

\begin{abstract}
The objective of the study is to assess the role of MMP-9, TIMP-1 \& 2 as non-invasive markers of liver fibrosis in the NAFLD. The 99 patients with NAFLD and different stages of fibrosis were examined. We assessed of anthropometric indicators, biochemical analysis of blood, abdominal ultrasonic studies, the levels of MMP-9, TIMP-1 \& 2. According of results of elastometry, patients were divided into five groups: $n=27, n=22, n=23, n=14, n=13$, respectively, depending on the stage of fibrosis ( $0 \sim 4$ ). Between the groups in physical examination, no significant differences in $B M I$ and $W / H$ were found. $64.6 \%$ of patients had abdominal obesity (BMI: 31.5 (29.1 33.9), $W / H$ : $1.02(1.01 \sim 1.05)$ ). Obesity and abdominal obesity ( $B M I$ and $W / H)$ had a significant positive relationship of moderate streight $\left(r_{s}=0.257, p<0.04\right.$ and $r_{s}=0.301, p<0.02$, respectively), with the stage of liver fibrosis. The groups were significant differences in the level of glucose, total bilirubin $(p<0.04, p<0.03$, respectively). No significant differences in the level of liver function tests $(A L T$ and $A S T)$ in the study groups were found. Significant differences were found in level of TIMP-2 $(p<0.04)$. TIMP-2 had a significant positive correlation with the severity of fibrosis in the hepatic tissue $\left(r_{s}=0.349\right.$, $p<0.004)$. TIMP-2 may be considered as a potential non-invasive marker for the diagnosis of liver fibrosis in patients with NAFLD.
\end{abstract}

Key words: NAFLD (nonalcoholic fatty liver disease), fibrosis, non-invasive diagnostic, matrix metalloproteinases, tissue inhibitors of metalloproteinases.

\section{Nomenclature}

NAFLD nonalcocholic fatty liver disease

MMP-9 matrix metalloproteinase 9

TIMP-1 \& 2 tissue inhibitor of matrix proteases $1 \& 2$

$B M I \quad$ body mass index

$W / H$ the ratio of waist to hip volume

NASH nonalcoholic steatohepatitis

T2DM type 2 diabetes mellitus

CVD cardiovascular disease

MS metabolic syndrome

ALT alanine aminotransferase

AST aspartate aminotransferase

GGT gamma-glutamyl transpeptidase

US ultrasound

WC waist circumference

HC hip circumference

$p \quad$ differences or reliability

$r_{s} \quad$ force Spearman correlation method

$n \quad$ sample number

\section{Introduction}

NAFLD ranks one of the leading positions among

Corresponding author: Tatyana Krolevets, postgraduate, research fields: gastroenterology and hepatology. diffuse liver diseases and it is getting widespread around the world [1]. The proportion of patients with NAFLD ranges from $6.3 \%$ to $33 \%$ with a median of $20 \%$ in the general population. According to clinical and histological changes, NAFLD is classified into several stages, such as steatosis, steatohepatitis, fibrosis and cirrhosis, which, upon the progression of disease, gradually interchange each other. Based on the data of the Russian Epidemiological Study DIREG L01903, the prevalence of NAFLD among adults in Russia has been $27 \%$, including $80.3 \%$ of hepatic steatosis, $16.8 \%$ cases for NASH and $2.9 \%$ for cirrhosis cases [2]. It is known that NAFLD is closely associated with obesity, overweight, insulin resistance, dyslipidemia and T2DM, the incidence of which has reached the epidemic proportions [3-5]. Moreover, the highest prevalence of CVD was determined in patients with NAFLD than in patients without NAFLD regardless of obesity and traditional cardiovascular risk factors. Thus, death rate, due to CVD compared 
with mortality rate from liver disease, is $7.5 \%$ and $0.2 \%$, respectively. In patients with steatosis and $\mathrm{NASH}$, these indices increase in four and more times, and death rate from CVD in NASH group prevails over mortality from liver diseases and reaches 36\% against $6.8 \%$, respectively $[1,6]$. Studying NAFLD is a burning issue due to the recent evidence that in a quarter of patients (27\%) fibrosis is developing within nine years and each fifth patient (19\%) has cirrhosis of the different stage of intensity [7]. In long-term observation, the progression of fibrosis is detected in $50 \%$ of patients with NAFLD [8], and the process often runs without clinical manifestations [9, 10]. Population studies are suggested that $60 \sim 80 \%$ cases of cryptogenic cirrhosis are NASH outcomes. NAFLD is at the top position among the causes for liver transplantation all around the world [11].

Therefore, the development of prognostic criteria for liver fibrosis in patients with NAFLD assumes a special actuality to prevent the progression of the disease in some cases and disability and mortality related thereto.

"The gold standard" for NAFLD diagnosis is considered the liver needle biopsy. Morphological examination allows determining the level of NASH activity and the stage of liver fibrosis [12-14]. Nowadays, a great attention is attended to the non-invasive methods for fibrosis diagnosis due to invasiveness, the significant inaccuracy of the histological studies related to the accuracy of puncture while the liver needle biopsy, the difference in results interpretation and the qualification of the doctor conducting the study. Thus, flexography (FibroScan) is a diagnostic method, determining the density (fibrosis) of the liver by the elastic waves. But we should remember that the sensitivity of this method reduces, when the $B M I$ is over 30 , an indicator of obesity, and it does not allow differentiating steatosis and steatohepatitis. Thus, bioprognostic laboratory tests with direct and indirect markers for liver fibrosis are assumed a paramount importance. The various calculated prognostic formula for assessment of fibrosis severity are suggested for clinical practice, such as APRI, ELF, NAFLD Fibrosis Score, FIB-4, FibroFast, FibroIndex, FibroMeter, FPI, Forns, GUCI, Hepascore, HALT-C, MDA, PGA, PGAA; however, the sensitivity and specificity of each formula are different and are not determined [15]. In particular, the information about MMP-9 and TIMP-1 \& 2 as the direct markers in pathogenesis, diagnosis and prognosis for liver fibrosis in NAFLD is limited and controversy $[16,17]$.

\section{Materials and Methods}

A number of 99 patients with NAFLD were examined. Inclusion criteria were age 18 65 years, the presence of NAFLD with different stage of fibrosis, signed informed consent. Exclusion criteria were suspected alcohol or drug abuse; medicamental, viral, autoimmune liver damage; storage diseases, hepatic cirrhosis, oncological diseases, severe diseases (uncorrected hypertension, T2DM in decompensation stage, chronic cardiac failure III-IV functional class, myocardium infarction and strokes in anamnesis), pregnancy, lactation period and low compliance.

All patients included into the study were asked concerning complaints, patient's life history, drug history, the presence of diseases associated with the MS. The general clinical examination was conducted including anthropometry with $B M I$ calculation, waist and hip measurement with $W / H$ ration counting, examination of skin and the abdominal organs, laboratory tests (general blood and urine tests, plasma glucose on the empty stomach, total protein, bilirubin, ALT, AST, alkaline phosphatase, GGT, total cholesterol and its fractions) and instrumental methods of examination, such as abdominal US. MMP-9 and its inhibitors (TIMP-1 \& 2) were detected for noninvasive evaluation of fibrosis stage. The patients were also performed the liver elastometry for assessment of liver fibrosis using METAVIR score with FibroScan. 
Results processing and graphical analysis were performed using PC with Microsoft Excel and STATISTICA 6.1 (Russian version) software and based on the recommendations of the leading authors in the field of statistical data processing [14-16]. Due to the fact that the distribution of parameters in groups was different from the normal figures, the data were presented as medians (interquartile range). Comparison of samples was performed using nonparametric tests: Kruskal-Wallis test $(H)$ for quantitative data and contingency tables ( $X^{2}$ criteria). Intercorrelation between the indicators was assessed using Spearman correlation analysis $\left(r_{s}\right)$. The correlation strength between the indicators in the the values of correlation coefficients from 0.0 to -0.25 and up to +0.25 was assessed as a lack of correlation or weak one; from 0.26 to 0.5 (from -0.26 to -0.5 ) as a moderate one; from 0.51 to 0.75 (from -0.5 to -0.75 ) as a mean one; and over $0.75(-0.75)$ as a strong correlation. At all stages of the statistical analysis the null hypothesis was rejected in $p$ values lower than 0.05 .

\section{Results and Discussion}

The cohort of patients with NAFLD was presented by female patients $(n=16)$ and middle-aged males (median age was 45 years, upper and lower quartiles were 40 and 55 years, respectively). Steatosis was diagnosed in $57.6 \%$ of these patients and steatohepatitis in $42.4 \%$, respectively.

The patients were divided into the following groups: Group 1 included the patients with zero stage of fibrosis $(n=27)$, Group 2 consisted of the patients with the Stage $1(n=22)$, Group 3 comprised of patients with the Stage $2(n=23)$, Group 4 was the Stage $3(n=14)$ and Group 5 included the patients with the Stage $4(n=13)$.

It is known that the pathognomonic clinical signs of NAFLD are not determined. It was revealed that a half of the patients had an asymptomatic course of the disease (43.4\%) with complaints of pyrosis, epigastric burning, pains in the epigastric region, and others. The rest patients also had complaints on pain in the right hypohondrium (34.4\%), a sense of discomfort in the right hypohondrium (29.3\%), bitter in the mouth (39.4\%). And $14.1 \%$ of patients had complaints on general weakness $(29.3 \%)$ and fatigability (28.3\%). There were no significant differences in frequency and intensity of these symptoms in all groups.

Analyzing the diseases and conditions in case of having metabolic syndrome, risk factors and NAFLD progression (obesity and overweight, hypertension, impaired glucose tolerance or type 2 diabetes, hyperand dyslipidemia, and others) no discrepancies were detected in the groups.

The groups as per examination results had no statistical differences in age, systolic and diastolic blood pressure, height, weight, BMI, waist and hip circumference (WC and $\mathrm{HC}$, respectively). Most patients had obesity (BMI median was $31.5 \mathrm{~kg} / \mathrm{m}^{2}$ and upper and lower quartiles were $29.1 \mathrm{~kg} / \mathrm{m}^{2}$ and 33.9 $\mathrm{kg} / \mathrm{m}^{2}$, respectively). Herewith, $43.4 \%$ of patients had obesity Class 1, and $15.2 \%$ and $4.04 \%$ of persons had obesity Class 2 and 3, respectively. $35.4 \%$ of people were overweight.

Genetic and non-genetic factors for liver fibrosis progression in NAFLD have been detected. Among the genetically stipulated factors, it was determined HFE (hemochromatosis gene), angiotensinogen and transforming growth factor- $\beta 1$ (TGF- $\beta 1$ ). Such factors as obesity, T2DM and hypertriglyceridemia have been considered as non-genetic factors [18]. Despite a lack of differences in the groups, the supplementary test was conducted to analyze the correlation between the stage of hepatic fibrosis and hypertension, T2DM, obesity degree as per $B M I$, the parameters of total cholesterol and triglycerides. Significant positive correlation was determined only with $B M I$ indices $\left(r_{s}=0.257, p<0.04\right)$ indicating the progression of fibrosis in patients with NAFLD while gaining the body weight.

According to the results of WC and $\mathrm{HC}$ 
measurement followed by counting $W / H$ indices, all patients were diagnosed the abdominal type of obesity ( $W / H$ median was 1.02; the upper and lower quartiles were 1.01 and 1.05 , respectively). Numerous studies have indicated on the bonds between NAFLD, MS and visceral obesity. Pathologic obesity in $95 \sim 100 \%$ of cases was combined with the development of hepatosis, and in $20 \sim 47 \%$ of cases with NASH $[19,20]$. About $25 \%$ of patients with NAFLD were not obese, but the laboratory and instrumental examinations gave evidences on the fatty changes in the liver as per the type of fatty hepatosis [13]. One study was reported that among patients with NAFLD, but without diabetes mellitus, $22 \%$ was thin, $64 \%$ did not meet the minimal criteria of MS, and $12 \%$ of patients was identified no MS criteria. Korean scientists Chang et al. [21] reported that liver steatosis was developing in patients with the increase of body weight, but without $B M I$ elevation [21].

Undoubtedly, $B M I$ is a simple, easily reproducible, reliable screening criterion for evaluation of normal weight, overweight and obesity. At the same time, recent studies have demonstrated that BMI has not been a sufficient criterion for prognosis of the development and course of all diseases associated with obesity and overweight. Thus, at the 23th Annual Congress in 2014, American Association of Endocrinologists considered a new diagnostic algorithm for obesity consisting of two components: (1) BMI assessment taking into consideration the ethnic features to identify the individuals with the increased amount of adipose tissue; (2) presence and severity of complications associated with obesity. Thuswise, today it has been noted the changeover from obesity assessment based on BMI (BMI-based approach) to obesity assessment as per the presence or absence of obesity-related diseases or disease states (complication-based approach). NAFLD was related to such diseases, and steatosis in combination with fibrosis and/or positive inflammatory tests to its complicated forms [22].
Upon conducting the correlation analysis between fibrosis stage and the nature of adipose tissue distribution $(\mathrm{WC}, W / H)$ it was registered the reliable significant positive correlation of moderate intensity $\left(r_{s}=0.304, p<0.02\right.$ and $r_{s}=0.301, p<0.02$, respectively), confirming the importance of fibrosis stage assessment in NAFLD in patients of the risk groups, suffering from abdominal obesity. According to the results of physical examination in $21.2 \%$ of patients, no clinically significant changes have been identified. More than half of patients (64.6\%) noticed painfulness while palpation of the inferior border of liver, the enlargement of liver was detected in $46.5 \%$ of cases. Furthermore, in patients with NAFLD and fibrosis 1 stage, these changes were statistically detected more frequently than in the group with the higher stage of fibrosis. The high prevalence of pain symptoms in the right subcostal area during examination of these patients may be stipulated by cholelithiasis. The close anatomical and functional association between the biliary system and gastroduodenal zone contributed to the formation and progression of pathological changes in these organs [23]. In NAFLD, latent or manifested changes of hepatocyte functions led to the formation of defective bile micelles with a high cholesterol level and the reduced content of phospholipids and bile acids, facilitating the bile lithogenicity and stimulating the development of cholelithiasis, which had the third place after cardiovascular diseases and diabetes, and may be secondary one at the background of metabolic disorders.

The results of the laboratory methods by groups are presented in Table 1. Significant differences in glucose and total bilirubin parameters were identified. The high incidence of impaired glucose tolerance $(20 \%)$ and T2DM (40\%) was registered. That is why NAFLD is considered as a precursor of carbohydrate metabolism disorders [2]. Liver in MS holds not only a prominent place in pathogenesis of insulin resistance, dyslipidemia and hyperglycemia, but also acts as a target 

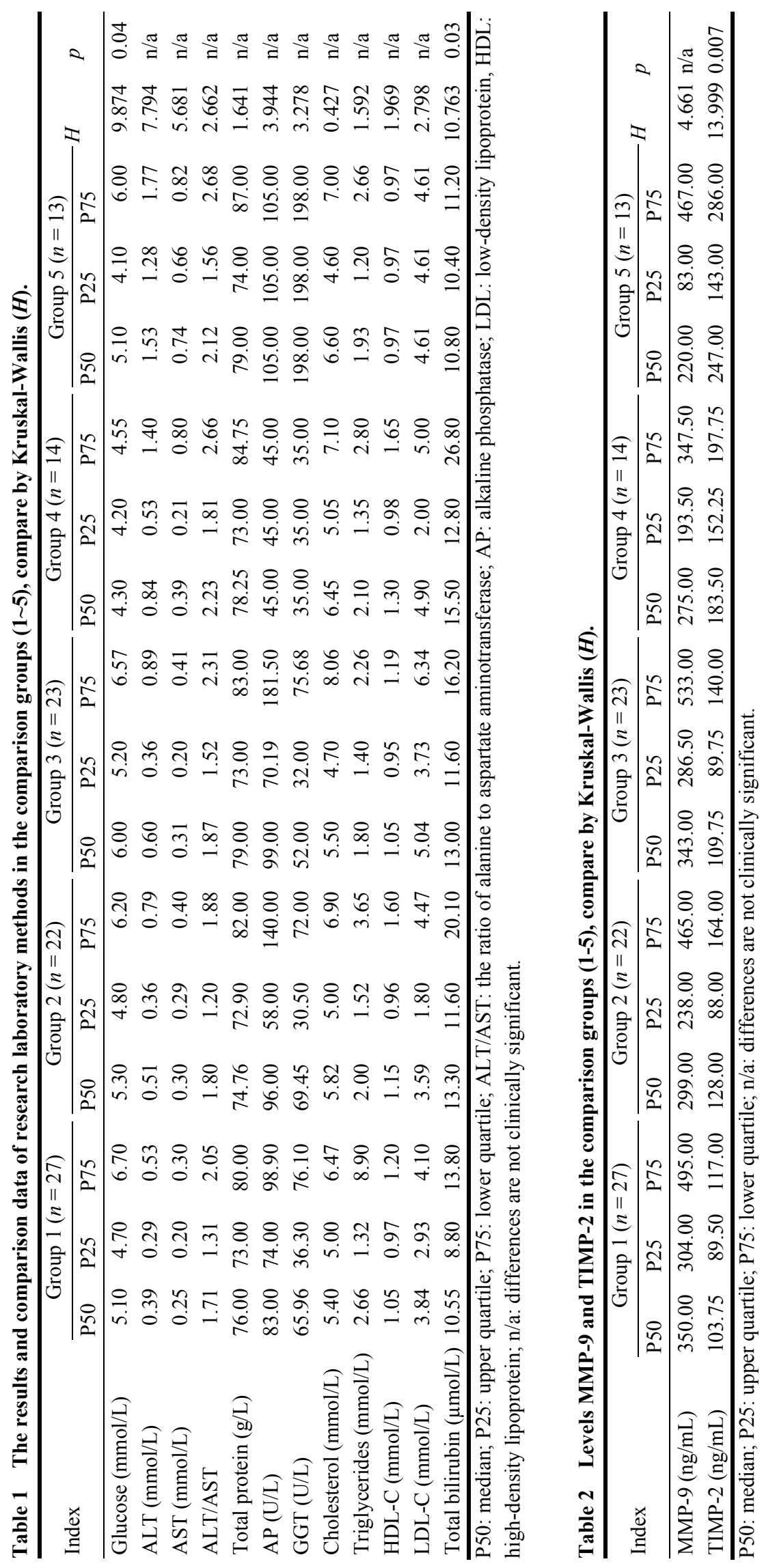


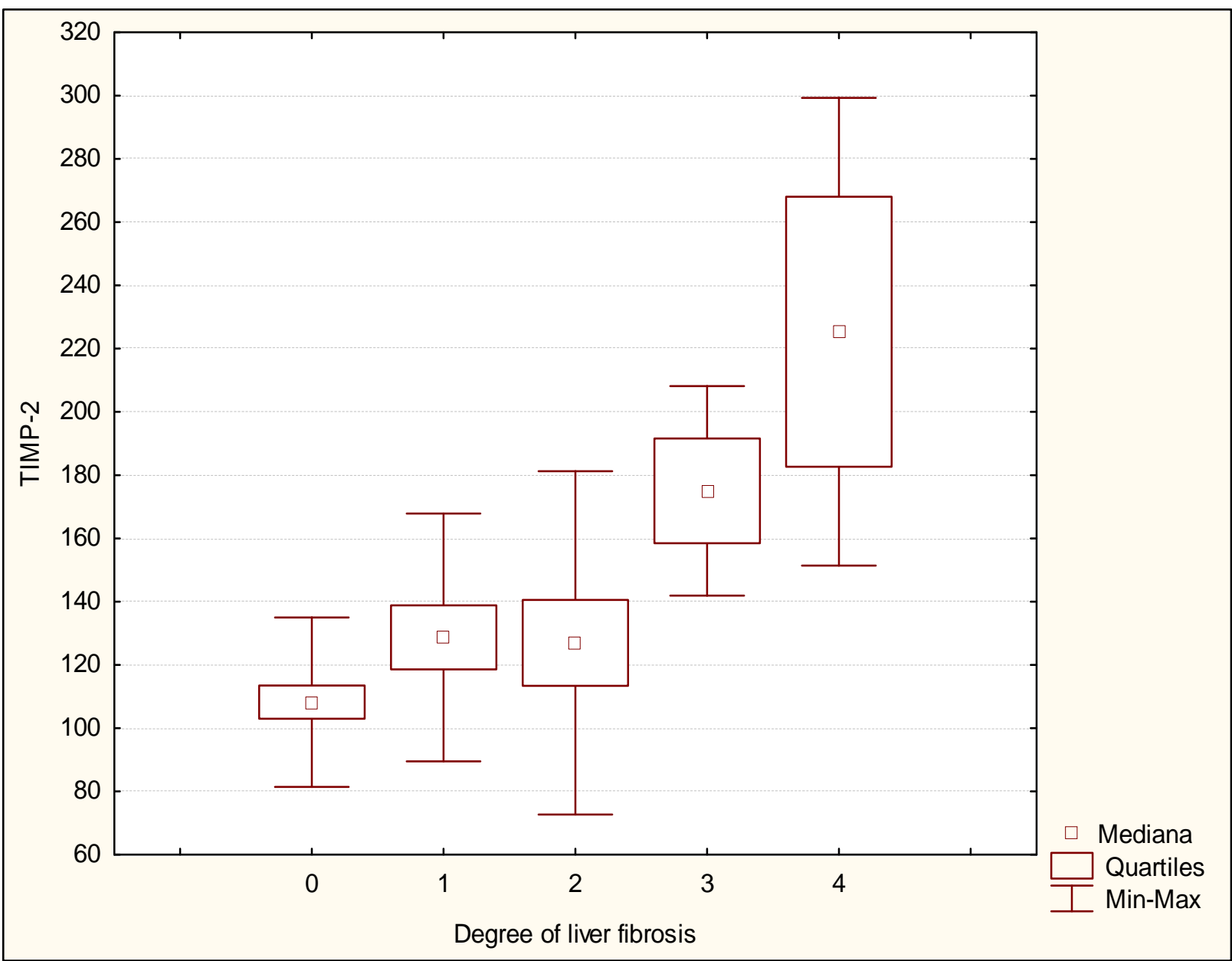

Fig. 1 Amplitude level diagram TIMP-2 depending on the extent of liver fibrosis (Groups 1-5).

organ [24]. In several studies, it was suggested to consider NAFLD as MS component [23, 25, 26]. However, there is no direct evidence of NAFLD among MS official criteria [27-29].

Interestingly, as per the results of the marker evaluation in blood, the cytolytic and cholestatic differences were not detected, but all groups demonstrated the high level of ALT $(0.45(0.33 \sim 0.76)$ $\mathrm{mol} / \mathrm{L}$ ) and ALT/AST ratio was over 1 (1.8 (1.4 2.2)), which was also considered as a predictor of the development of liver fibrosis in NAFLD. However, high level of serum transaminases in analysis of literature data was revealed only in 20 21\% of patients with NAFLD and their normal values did not exclude the probability of necrotic-inflammatory changes existence and liver fibrosis [30]. In this regards, it has been interesting to study the non-invasive biomarkers of liver fibrosis.

The results of these calculations are presented in Table 2. Significant differences were found only on TIMP-2 level. The increase values of this indicator were noted in case of increasing of fibrosis severity, which is indicated in Fig. 1. TIMP-2 also had a highly reliable significant positive correlation of moderate intensity with the severity of fibrosis in the liver tissue $\left(r_{s}=0.349, p<0.004\right)$.

\section{Conclusions}

According to the results of our study, it was determined a high prevalence of NAFLD in patients of the senior age group and the tendency to asymptomatic disease course. It was registered the 
clinical markers of liver fibrosis progression, such as obesity with abdominal adipose tissue distribution. TIMP-2 is considered as a potential noninvasive marker for liver fibrosis diagnosis in patients with NAFLD.

\section{Acknowledgments}

This study was supported by Russian Federation, Omsk, State Educational Institution of Higher Vocational Education "Omsk State Medical University" and by grand named "Prognostic Model for Non-invasive Assessment of Liver Fibrosis in Patients with Different Degrees of Non-alcoholic Fatty Liver Disease" as part of the state task under the heading "Implementation of Research and Development" on the instructions of Russian Ministry of Health in the year 2015 2017.

\section{Conflict of interests}

The authors declared no conflict of interests in relation to this manuscript.

\section{References}

[1] World Gastroenterology Organization. 2012. "Nonalcoholic Fatty Liver Disease and Nonalcoholic Steatohepatitis: World Gastroenterology Organisation Global Guidelines.” World Gastroenterology Organisation. Accessed December 1, 2012. http://www.worldgastroenterology.org/NAFLD-NASH.ht $\mathrm{ml}$.

[2] Drapkina, O. M., and Ivashkin, V. T. 2014. "Epidemiologic Features of Non-alcoholic Fatty Liver Disease in Russia (Results of Open Multicenter Prospective Observational Study DIREG L 01903)." Russian Journal of Gastroenterology and Hepatology, Coloproctology 24 (4): 32-8.

[3] World Gastroenterology Organization. 2009. "Obesity: World Gastroenterology Organization Global Guideline.” World Gastroenterology Organization. Accessed December 1, 2012. http://www.worldgastroenterology.or g/obesity.html.

[4] Qureshi, K., and Abrams, G. A. 2007. "Metabolic Liver Disease of Obesity and Role of Adipose Tissue in the Pathogenesis of Nonalcocholic Fatty Liver Disease." World J. Gastroenterol 13: 3540-53.

[5] Utzschneider, K. M., and Kahn, S. E. 2006. "Review: The
Role of Insulin Resistance in Nonalcocholic Fatty Liver Disease." J. Clin. Endocrinol. Metab. 91: 4753-61.

[6] Bellentani, S., Bedogni, G., and Tiribelli, C. 2008. "Liver and Heart: A New Link?.” J. Hepatol. 49: 300-2.

[7] Reynaert, H., Geerts, A., and Henrion, J. 2005. "The Treatment of Non-alcoholic Steatohepatitis with Thiazolidinediones." Aliment. Pharmacol. Ther. 22 (10): 897-905.

[8] Bacon, B. R. 2000. "Clinical Course and Prognosis of Non-alcoholic Steatohepatitis (NASH)." Presented at Falk Symposium Steatohepatitis, Den Haag.

[9] Fedorov, I. G., Nikitin, I. G., and Storozhakov, G. I. 2004. "Nealkogol'nyi steatogepatit: klinika, patogenez, diagnostika, lechenie." Consilium medicum 6 (6): 401-5.

[10] Khazanov, A. I. 2005. "Vozmozhnosti progressirovaniya alkogol'nogo i nealkogol'nogo steatogepatita $\mathrm{v}$ tsirroz pecheni." Ros. Zhurn. Gastroenterol. Gepatol. Koloproktol. 15 (2): 26-32.

[11] Clark, J. M. 2003. "The Prevalense and Etiology of Elevated Aminotransferase Levels in the United States." Am. J. Gastroenterol. 98: 955-6.

[12] Marchesini, G., Bugianesi, E., Forlani, G., Cerrelli, F., Lenzi, M., Manini, R., et al. 2003. "Nonalcoholic Fatty Liver, Steatohepatitis, and the Metabolic Syndrome." Hepatology 37: 917-23.

[13] Bogomolov, P. O., and Tsodikov, G. V. 2006. "Nealkogol'naya zhirovaya bolezn' pecheni." Spravochnik Poliklinicheskogo Vracha 4 (1): 2.

[14] Kleiner, D. E., Brunt, E. M., Van Natta, M., Behling, C., Contos, M. J., Cummings, O. W., et al. 2005. "Nonalcoholic Steatohepatitis Clinical Research Network. Design and Validation of a Histological Scoring System for Nonalcoholic Fatty Liver Disease." Hepatology 41: 1313-21.

[15] Livzan, M. A., Korolevets, T. S., Lapteva, I. V., and Cherkashchenko, N. A. 2014. "Non-alcoholic Fatty Liver Disease in the Patients Presenting with Abdominal Obesity." Evidence Gastroenterology 4: 8-14.

[16] Hemmann, S., Graf, J., Roderfeld, M., and Roeb, E. 2007. "Expression of MMPs and TIMPs in Liver Fibrosis-A Systematic Review with Special Emphasis on Anti-fibrotic Strategies." Journal of Hepatology 46: 955-75.

[17] Lim, J. W., Dillon, J., and Miller, M. 2014. "Proteomic and Genomic Studies of Non-alcocholic Fatty Liver Disease-Clues in the Pathogenesis." World Journal of Gastroenterology 20 (26): 8325-40.

[18] Bataller, R., and Brenner, D. A. 2005. "Liver Fibrosis." $J$. Clin. Invest. 115 (2): 209-18.

[19] Yu, A. S., and Keeffe, E. B. 2002. "Nonalcoholic Fatty Liver Disease." Gastroenterol. Dis. 2 (1): 11-9.

[20] Livzan, M. A., Korolevets, T. S., Lapteva, I. V., and 
Cherkashchenko, N. A. 2015. "Leptin Resistance in Patients with Nonalcoholic Fatty Liver Disease Associated with Obesity and Overweight." Medical Advice 13: 58-62.

[21] Chang, Y., Ryu, S., Sung, E., Woo, H. Y., Cho, S. I., Yoo, S. H., et al. 2009. "Weight Gain within the Normal Weight Range Predicts Ultrasonographically Detected Fatty Liver in Healthy Korean Men.” Gut. 58: 1419-25.

[22] Samorodskaya, I. V. 2014. "The New Paradigm of Obesity." Therapist 12: 10-5.

[23] Lazebnik, L. B., Zvenigorodskaya L. A., and Egorova, E. G. 2005. "Metabolicheskii sindrom s pozitsii gastroenterologa." Rus. Med. Zhurn. 13 (26): 1706-20.

[24] Zilov, A. V. 2005. 'Pechen' pri metabolicheskom sindrome i insulinorezistentnosti: vzglyad endokrinologa." Klin. Perspektivy Gastroenterol. Gepatol. 5: 13-7.

[25] Korneeva, O. N., Drapkina, O. M., Bueverov, A. O., Ivashkin, V. T. 2005. "Nealkogol'naya zhirovaya bolezn' pecheni kak proyavlenie metabolicheskogo sindroma."
Klin. Perspektivy Gastroenterol. Gepatol. 4: 21-4.

[26] Mansurov, K. K., Mirodzhov, G. K., and Mansurova, F. K. 2005. 'i dr. Insulinorezistentnost' u bol'nykh metabolicheskim sindromom i zhelchnokamennoi bolezn'yu." Klinicheskaya meditsina 83 (7): 48-51.

[27] Alberti, K. G., and Zimmet, P. Z. 1998. "Definition, Diagnosis, and Classification of Diabetes Mellitus and Its Complications, Part I: Diagnosis and Classification of Diabetes Mellitus: Provisional Report of a WHO Consultation." Diabet. Med. 15: 539-53.

[28] American Association of Clinical Endocrinologists. 2002. "Medical Guidelines for the Management of Diabetes Mellitus: The AACE System of Intensive Diabetes Self-Management." Endocrine Pract. 8: 40-82.

[29] Reaven, G. M. 1988. "Role of Insulin Resistance in Human Disease." Diabetes 37 (12): 1595-607.

[30] Medina, J., Fernández-Salazar, L. I., García-Buey, L., and Moreno-Otero, R. 2004. "Approach to the Pathogenesis and Treatment of Nonalcoholic Steatohepatitis." Diabetes Care 27 (8): 2057-66. 